\title{
The Simulation of a Queueing System Consist of Two Parallel Heterogeneous Channels with no Waiting Line
}

\author{
Vedat Sağlam ${ }^{1, ~ *, ~ M u r a t ~ S a g ̆ ı r ~}{ }^{1}$, Erdinç Yücesoy ${ }^{1}$, Müjgan Zobu ${ }^{2}$ \\ ${ }^{1}$ Department of Statistics, Ondokuz Mayıs University, Samsun, Turkey \\ ${ }^{2}$ Department of Statistics, Amasya University, Amasya, Turkey
}

Email address:

vsaglam@omu.edu.tr (V. Sağlam)

To cite this article:

Vedat Sağlam, Murat Sağır, Erdinç Yücesoy, Müjgan Zobu. The Simulation of a Queueing System Consist of Two Parallel Heterogeneous Channels with no Waiting Line. Pure and Applied Mathematics Journal. Vol. 4, No. 5, 2015, pp. 216-218. doi: 10.11648/j.pamj.20150405.12

\begin{abstract}
A queueing system with two parallel heterogenous channels without waiting is considered. In this queueing system customer arrivals are Poisson distributed with $\lambda$ rate. Each customer has exponentially distributed service time with $\mu_{k}(k=$ $1,2)$ parameter at $k-t h$ channel. When a customer arrives this system if both the service channels are available, the customer has service with $\alpha$ or $\beta=1-\alpha$ probabilities at first and second service channels respectively. If one of the service channels is available, the customer has service at this service channel or leaves the system without being served if both of the service channels are busy. We have obtained mean waiting time and mean number of customers of the system and a simulation of this system is performed.
\end{abstract}

Keywords: Heterogeneous Queueing Systems, Loss Probability, Optimization, Simulation, Markov Chain, Kolmogorov Equation, Transition Rates

\section{Introduction}

Stochastic queueing models and their applications have important roles in fields such as transportation, computer networks, communication, production lines and etc. There are a lot of studies regarding queueing systems with homogeneous Poisson arrivals. In fact in real life practical applications the arrivals to queueing systems are non-homogeneous. The mean customer number and the distribution of waiting time in a tandem queueing system with Poisson arrivals are obtained in [1]. The response time of a tandem Markovian queue with blocking is studied in [2]. A heterogeneous stochastic queueing system with two channel in which no waiting is allowed is analyzed by [3]. Performance measures of a tandem queueing system with two stages are obtained and these performance measures are given in [4]. A queueing model with non-homogeneous bulk arrivals under parallel and series configuration is developed and analyzed in [5]. A number of different models for Markov-modulated queueing systems surveyed. Then a model in which the workload process and the secondary process together constitute a Markov compound Poisson process is analyzed by [6]. In [7] a tandem queueing system that can be formulated as a continuous-time Markov chain is considered and this system is investigated how to maximize the throughput when the queue is considered and several monotonicity properties of optimal policies for such a system are proved in [8]. The algorithms for the Multi-Server Queue is had in [12]. A queueing system with two parallel heterogeneous channels without waiting is considered in [9], in this system the arrivals are Poisson distributed with $\lambda$ rate. Each customer has exponentially distributed service time with $\mu_{k}(k=1,2)$ parameter at $k-t h$ channel. When a customer arrives this system if both the service channels are available, the customer has service with $\alpha$ or $\beta=1-\alpha$ probabilities at first and second service channels respectively. If one of the service channels is available, the customer has service at this service channel or leaves the system without being served if both of the service channels are busy so loss occurs. This loss probability optimized in [9]. In this paper we obtain mean waiting time and mean number of customers of the system studied in [9]. In addition, the mean waiting time of this system is optimized under customized conditions. Finally a 100.000 step simulation is done for this system and it is seen that the simulation results tend to exact results.

\section{Definition of the Queueing System}

The customers arrive this queueing system according to Poisson distribution with $\lambda$ parameter. In $k-t h$ service, each customer has exponentially distributed service time with 
$\mu_{k}(k=1,2)$ service parameter. When a customer arrives this system if both the services are available the customer has service with $\alpha$ and $\beta=1-\alpha$ probabilities at first and second service channels respectively. If one of the service channel is available the customer has service at this service channel or leaves the system without being served if both of the service channels are busy so loss occurs. The system is shown in Figure 1 below.

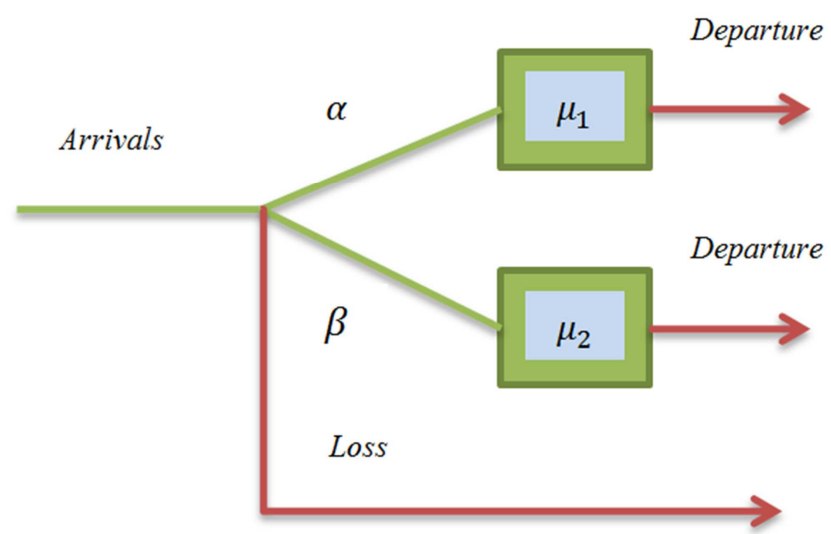

Figure 1. A heterogeneous queueing system with parallel channels without waiting line.

\section{Kolmogorov Equations}

Transient Solutions in Markovian queueing systems are obtained in [13]. Two dimensional $P_{i j}(t)$ Markov chain defines the probability that there are $i$ customers at first service channel and there are $j$ customers at second service channel at a given time $t$. Where $\forall i, j \in\{0,1\}$. The Kolmogorov differential equations of these transient probabilities are obtained as below:

$P_{00}^{\prime}(t)=-\lambda P_{00}(t)+\mu_{1} P_{10}(t)+\mu_{2} P_{01}(t)$

$P_{10}^{\prime}(t)=-\left(\lambda+\mu_{1}\right) P_{10}(t)+\alpha \lambda P_{00}(t)+\mu_{2} P_{11}(t)$

$P_{01}^{\prime}(t)=-\left(\lambda+\mu_{2}\right) P_{01}(t)+\beta \lambda P_{00}(t)+\mu_{1} P_{11}(t)$

$P_{11}^{\prime}(t)=-\left(\mu_{1}+\mu_{2}\right) P_{11}(t)+\lambda\left(P_{01}(t)+P_{10}(t)\right)$

where

$$
P_{i j}^{\prime}(t)=\sum P_{i k}(t) a_{k j}
$$

The transition rates matrices $\Lambda=\left[a_{i j}\right]$ is:

$$
\Lambda=\left[\begin{array}{cccc}
-\lambda & \mu_{1} & \mu_{2} & 0 \\
\alpha \lambda & -\left(\lambda+\mu_{1}\right) & 0 & \mu_{2} \\
\beta \lambda & 0 & -\left(\lambda+\mu_{2}\right) & \mu_{1} \\
0 & \lambda & \lambda & -\left(\mu_{1}+\mu_{2}\right)
\end{array}\right]
$$

in which $a_{i j} \geq 0$ and $a_{i i}<0$ and $\sum_{j} a_{i j}=0$

Transient probabilities of the system above are found as:

$$
\begin{aligned}
& P_{00}=\frac{\mu_{1} \mu_{2}\left(2 \lambda+\mu_{1}+\mu_{2}\right)}{\mu_{1} \mu_{2}\left(2 \lambda+\mu_{1}+\mu_{2}\right)+\left[\lambda\left(\mu_{1}+\mu_{2}\right)+\lambda^{2}\right]\left[\lambda+\alpha \mu_{2}+\beta \mu_{1}\right]} \\
& P_{10}=\frac{\lambda \mu_{2}\left(\lambda+\alpha\left(\mu_{1}+\mu_{2}\right)\right)}{\mu_{1} \mu_{2}\left(2 \lambda+\mu_{1}+\mu_{2}\right)+\left[\lambda\left(\mu_{1}+\mu_{2}\right)+\lambda^{2}\right]\left[\lambda+\alpha \mu_{2}+\beta \mu_{1}\right]}
\end{aligned}
$$

$$
\begin{aligned}
& P_{01}=\frac{\lambda \mu_{1}\left(\lambda+\beta\left(\mu_{1}+\mu_{2}\right)\right)}{\mu_{1} \mu_{2}\left(2 \lambda+\mu_{1}+\mu_{2}\right)+\left[\lambda\left(\mu_{1}+\mu_{2}\right)+\lambda^{2}\right]\left[\lambda+\alpha \mu_{2}+\beta \mu_{1}\right]} \\
& P_{11}=\frac{\lambda^{2}\left(\lambda+\alpha \mu_{2}+\beta \mu_{1}\right)}{\mu_{1} \mu_{2}\left(2 \lambda+\mu_{1}+\mu_{2}\right)+\left[\lambda\left(\mu_{1}+\mu_{2}\right)+\lambda^{2}\right]\left[\lambda+\alpha \mu_{2}+\beta \mu_{1}\right]}
\end{aligned}
$$

Erlang B formula is given in $[10,11,14]$. The loss probability in queuing systems with heterogeneous servers is shown by $[15,16]$. Where, $P_{00}$ is the probability that the system is empty and $P_{11}$ is the probability that first and second service channels are busy also this is the loss probability. The optimization of loss probability is as follows:

When $\mu_{1}>\mu_{2}$ and $\alpha=1$ then loss probability is minimum [9].

When $\alpha=\beta=1 / 2$ under condition $\mu_{1}+\mu_{2}=c$ the loss probability $P_{2}\left(\mu_{1}, \mu_{2}\right)$ takes its minimum value for $\mu_{1}=$ $\mu_{2}=c / 2$.

$$
\min P_{2}=\frac{2 \lambda^{2}}{c^{2}+2 \lambda c+2 \lambda^{2}}
$$

as a result homogeneous system is better than heterogeneous system for $P_{2}\left(\mu_{1}, \mu_{2}\right)$ criteria [9].

\section{Performance Measures of System}

Performance measure of since there is no waiting in this queueing system, the mean customer number and the mean waiting time in the system is found.

Mean customer number in the system

$$
\begin{gathered}
E(N)=\sum_{i, j}(i+j) P_{i j}= \\
\frac{\lambda\left(\mu_{1}+\mu_{2}\right)\left(\lambda+\alpha \mu_{2}+\beta \mu_{1}\right)+2 \lambda^{2}\left(\lambda+\alpha \mu_{2}+\beta \mu_{1}\right)}{\mu_{1} \mu_{2}\left(2 \lambda+\mu_{1}+\mu_{2}\right)+\left[\lambda\left(\mu_{1}+\mu_{2}\right)+\lambda^{2}\right]\left[\lambda+\alpha \mu_{2}+\beta \mu_{1}\right]}
\end{gathered}
$$

Mean waiting number in the system and its optimization

$$
E(T)=\frac{\alpha}{\mu_{1}}+\frac{\beta}{\mu_{2}}
$$

Similarly, optimizations for mean waiting time in the system $E(T)$ can be done. The optimizations done for loss probability above are also valid for $E(T)$.

When $\mu_{1}>\mu_{2}$ and $\alpha=1$, the loss probability $E(T)$ is minimum.

$$
\begin{gathered}
0>\mu_{2}-\mu_{1} \\
0>\alpha\left(\mu_{2}-\mu_{1}\right) \geq\left(\mu_{2}-\mu_{1}\right) \\
\frac{\mu_{1}+\alpha\left(\mu_{2}-\mu_{1}\right)}{\mu_{1} \mu_{2}} \geq \frac{\mu_{1}+\left(\mu_{2}-\mu_{1}\right)}{\mu_{1} \mu_{2}} \\
E(T) \geq \frac{\mu_{1}+\left(\mu_{2}-\mu_{1}\right)}{\mu_{1} \mu_{2}} \\
E(T) \geq \frac{1}{\mu_{1}}
\end{gathered}
$$

For $\alpha=\beta=1 / 2$ and under condition $\mu_{1}+\mu_{2}=c$, the loss probability $P_{2}\left(\mu_{1}, \mu_{2}\right)$ has its minimum value for $\mu_{1}=\mu_{2}=c / 2$.

$$
E(T)=\frac{1 / 2}{\mu_{1}}+\frac{1 / 2}{\mu_{2}}
$$




$$
\begin{aligned}
& E(T)=\frac{1}{2}\left(\frac{\mu_{1}+\mu_{2}}{\mu_{1} \mu_{2}}\right) \\
& E(T)=\frac{1}{2}\left(\frac{c}{\mu_{1} \mu_{2}}\right)
\end{aligned}
$$

To make $E(T)$ minimum, $\mu_{1} \mu_{2}$ product must be maximum. Under condition $\mu_{1}+\mu_{2}=c, \mu_{1} \mu_{2}$ product is maximum for $\mu_{1}=\mu_{2}=c / 2$.

$$
\min E(T)=\frac{1}{2}\left(\frac{4}{c}\right)=\frac{2}{c}
$$

\section{Simulation}

Table 1. Mean waiting time in the system and loss Probability for $\lambda=$ $0.63, \mu_{1}=0.94, \mu_{2}=1.46, \alpha=0.00, \beta=1.00$.

\begin{tabular}{lcccc}
\hline Iteration & \multicolumn{3}{c}{ Simulation Value } & \multicolumn{3}{c}{ Exact Value } \\
\cline { 2 - 5 } Numbers & $\boldsymbol{P}_{\boldsymbol{L}}$ & $\boldsymbol{E}(\boldsymbol{T})$ & $\boldsymbol{P}_{\boldsymbol{L}}$ & $\boldsymbol{E}(\boldsymbol{T})$ \\
\hline 500 & 0.0620 & 0.6579 & 0,07770 & 0,68493 \\
1000 & 0.0540 & 0.6790 & 0,07770 & 0,68493 \\
5000 & 0.0606 & 0.7034 & 0,07770 & 0,68493 \\
10000 & 0.0590 & 0.6885 & 0,07770 & 0,68493 \\
\hline
\end{tabular}

Table 2. Mean waiting time in the system and loss Probability for $\lambda=$ $0.63, \mu_{1}=0.94, \mu_{2}=1.46, \alpha=0.30, \beta=0.70$.

\begin{tabular}{lcccc}
\hline Iteration & \multicolumn{3}{l}{ Simulation Value } & \multicolumn{2}{c}{ Exact Value } \\
\cline { 2 - 5 } Numbers & $\boldsymbol{P}_{\boldsymbol{L}}$ & $\boldsymbol{E}(\boldsymbol{T})$ & $\boldsymbol{P}_{\boldsymbol{L}}$ & $\boldsymbol{E}(\boldsymbol{T})$ \\
\hline 500 & 0.0820 & 0.8219 & 0,08236 & 0,79860 \\
1000 & 0.0810 & 0.8012 & 0,08236 & 0,79860 \\
5000 & 0.0836 & 0.7942 & 0,08236 & 0,79860 \\
10000 & 0.0718 & 0.7955 & 0,08236 & 0,79860 \\
\hline
\end{tabular}

Table 3. Mean waiting time in the system and loss Probability for $\lambda=$ $0.63, \mu_{1}=0.94, \mu_{2}=1.46, \alpha=0.50, \beta=0.50$.

\begin{tabular}{lcccc}
\hline Iteration & \multicolumn{3}{c}{ Simulation Value } & \multicolumn{3}{c}{ Exact Value } \\
\cline { 2 - 5 } Numbers & $\boldsymbol{P}_{\boldsymbol{L}}$ & $\boldsymbol{E}(\boldsymbol{T})$ & $\boldsymbol{P}_{\boldsymbol{L}}$ & $\boldsymbol{E}(\boldsymbol{T})$ \\
\hline 500 & 0.0860 & 0.8571 & 0,08529 & 0,87438 \\
1000 & 0.0850 & 0.8564 & 0,08529 & 0,87438 \\
5000 & 0.0824 & 0.8504 & 0,08529 & 0,87438 \\
10000 & 0.0858 & 0.8422 & 0,08529 & 0,87438 \\
\hline
\end{tabular}

Table 4. Mean waiting time in the system and loss probability for $\lambda=$ $0.63, \mu_{1}=1.20, \mu_{2}=1.20, \alpha=0.50, \beta=0.50$.

\begin{tabular}{lcccc}
\hline Iteration & \multicolumn{3}{c}{ Simulation Value } & \multicolumn{3}{c}{ Exact Value } \\
\cline { 2 - 5 } Numbers & $\boldsymbol{P}_{\boldsymbol{L}}$ & $\boldsymbol{E}(\boldsymbol{T})$ & $\boldsymbol{P}_{\boldsymbol{L}}$ & $\boldsymbol{E}(\boldsymbol{T})$ \\
\hline 500 & 0.0820 & 0.8289 & 0,08288 & 0,83333 \\
1000 & 0.0820 & 0.8217 & 0,08288 & 0,83333 \\
5000 & 0.0828 & 0.8383 & 0,08288 & 0,83333 \\
10000 & 0.0824 & 0.8304 & 0,08288 & 0,83333 \\
\hline
\end{tabular}

\section{Conclusion}

Performance measures, the mean waiting time and the mean number of customers are obtained in this paper. In addition the optimization of mean waiting time is done under conditions given as $\mu_{1}>\mu_{2}, \alpha=1$, the loss probability and for $\alpha=\beta=1 / 2$ and under condition $\mu_{1}+\mu_{2}=c$.

Loss probability and mean waiting time in this system is obtained by $500,1000,5000$ and 10000 iteration steps and these results are given in Table1, Table2, Table 3 and Table4 respectively. Then it is seen that these simulation results tend to exact values. The number of service channels in the system can be adjusted or a waiting line can be added to this system for further studies.

\section{References}

[1] Jackson, R. R. P., "Queueing systems with Phase-Type Service", Operat. Res. Quart, 5, 109-120, 1954.

[2] J. R. Artalejo and G. Choudhury, "Steady State Analysis of an M/G/1 Queue with Repeated Attempts and Two-Phase Service", Quality Technology\&Quantative Management, Vol.1, No.2, pp. 189-199, 2004.

[3] M. Zobu, V. Sağlam, M. Sağır, E. Yücesoy and T. Zaman, "The Simulation and Minimization of Loss Probability in the Tandem Queueing with two Heterogeneous Channel", Mathematical problems in Engineering, Vol. 2013, Article ID 529010, 4 pages, 2013.

[4] V. Sağlam and M. Zobu, "A two-stage Queueing Model with No waiting Line between Channels", Mathematical Problems in Engineering, Vol. 2013, Article ID 679369, 5 pages, 2013.

[5] A. V. S. Suhasini, K. Srinivasa Rao, P. R. S. Reddy, "On parallel and series non homogeneous bulk arrival queueing model", OPSEARCH (Oct-Dec 2013), 50(4):521-547, 2013.

[6] N. U. Prabhu and Yixin ZHU, "Markov-modulated Queueing Systems”, Queueing System, 5 (1989) 215-246, 1989.

[7] Frederick S. Hillier, Kut C. So, "On the optimal design of tandem queueing systems with finite buffers", Queueing Systems 21 (1995) 245-266, 1995.

[8] V. V. Rykov, "Monotone Control of Queueing Systems with Heterogeneous Servers", Queueing Systems, 37, 391-403, 2001.

[9] V. Sağlam and H. Torun, “ On Optimization of Stochastic Service System with Two Heterogeneous Channels", International Journal of Applied Mathematics, 17, 1, 8 / 2005

[10] Gross, D., Harris, C. M., Thompson, M. J., Shortle, F. J., Fundementals of Queueing Theory, $4^{\text {th }}$ ed., John Wiley \& Sons, New York, 2008.

[11] Stewart, W. J., Probability, Markov Chains, Queues and Simulation, Princeton University Press, United Kingdom, 2009.

[12] V. Ramaswami, "Algorithms for the Multi-Server Queue", Commun. Statist.-Stochastic Models, 1(3), 393-417, 1985.

[13] W. K. Grassmann, “Transient Solutions in Markovian queueing systems", Computers \& Operations Research, Volume 4, Issue 1, pages 47-53, 1977.

[14] E. Brockmeyer, H. L. Halstrom and A. Jensen, "The life and works of A. K. Erlann.” Danish Acad. Techn. Sci, No. 2, Koben havn (Denmark), 1948.

[15] F. Alpaslan, "On the minimization probability of loss in queue two heterogeneous channels," Pure and Applied Mathematika Sciences, vol. 43, no. 1-2, pp. 21-25, 1996.

[16] V. Saglam and A. Shahbazov, "Minimizing loss probability in queuing systems with heterogeneous servers," Iranian Journal of Science and Technology, vol. 31, no. 2, pp. 199-206, 2007. 